DOI: http://dx.doi.org/10.20535/2219-380415201690855

В. В. Бурнашев ${ }^{1}$, доцент, к.т.н., С. М. Заика ${ }^{2}$, бакалавр

\title{
СИСТЕМА АВТОМАТИЧЕСКОГО УПРАВЛЕНИЯ СНАРЯДОМ НА ЭТАПЕ ВХОЖДЕНИЯ В ЛАЗЕРНЫЙ ЛУЧ
}

Nn The problem of the barrel guided missile entering a laser beam in the conditions of wind disturbances influence is considered. The mathematical model of motion is reduced, and balance motion parameters of the rocket are received. The simulation model of the shell is made.

The autonomous control system of the shell throughout the first second after ejection from the starting container is developed. Flight control laws are received by a method of optimum regulators analytical designing. Maximum wind velocity at which the autonomous control system can bring the shell in a beam depends on their structure. Angles of orientation and corresponding angular rates, a trajectory declination angle and a path angle, a velocity, and also linear coordinates of the shell can form the control laws. Thus it is determined, that the basic element of the shell control system at the entering stage in a laser beam should be strapdown inertial navigational system. Simplification of system sensors structure to an attitude system considerably reduces admissible wind speed at start. Control transfer from autonomous sys-

\footnotetext{
${ }^{1}$ Национальный технический университет Украины «Киевский политехнический институт», кафедра приборов и систем управления летательными аппаратами

${ }^{2}$ Национальный технический университет Украинь «Киевский политехнический институт», факультет авиационных и космических систем
} 
tem to laser-beam system happens smoothly under specially developed formula.

The search algorithm of a barrel installation angle is developed. Criterion of search is the error minimum on an altitude and a vertical velocity at limitations of a pitch and an altitude maximum of the shell subsidence. The search algorithm uses the method of full search. Thus the influence of a lateral and vertical wind on a shell flight path is considered.

Ua Розроблена автономна сучасна система керування стольною ракетою, якою можно керувати протягом першої секунди після сходження з пускового контейнера та забезпечити ії попадання в лазерний луч під дією вітра. Виконано імітаційне моделювання польоту снаряда. Визначена максимальна допустима швидкість вітру, при якій снаряд попадає в луч. Розроблено алгоритм пошуку кута встановлення ствола.

\section{Введение}

Современные ствольные управляемые ракеты второго поколения управляются с помощью лазерно-лучевой системы, которая обеспечивает высокую вероятность поражения целей [1].

Однако на протяжении первой секунды полета лазерный луч отсутствует, и ракета остается неуправляемой. В случае заваливания носовой части снаряда либо наличия боковых ветровых порывов может произойти его отклонение от желаемой траектории полета за пределы лазерного луча. Это повышает вероятность неудачных пусков и накладывает погодные ограничения на эксплуатацию изделия. Решение задачи управления снарядом на заданной траектории до попадания в лазерный луч в литературных источниках отсутствует.

\section{Постановка задачи}

Рассмотрим задачу синтеза автономной системы управления снарядом, действующей с момента вылета из ствола и способной обеспечить успешное попадание в лазерный луч в условиях влияния ветра.

\section{Математическая модель движения снаряда}

Движение управляемой ракеты достаточно точно можно описать с помощью системы уравнений [2]: 
$m \cdot \dot{V}_{a}=P \cdot \cos \alpha \cdot \cos \beta-X_{a}-G \cdot \sin \vartheta_{a}$

$m \cdot V_{a} \cdot\left(\dot{\vartheta}_{a} \cos \gamma_{a}-\dot{\psi}_{a} \cdot \cos \vartheta_{a} \sin \gamma_{a}\right)=Y_{a}+P \sin \alpha-G \cos \vartheta_{a} \sin \gamma_{a} ;$

$\dot{\omega}_{z} I_{z}=M_{z}-\left(I_{y}-I_{x}\right) \cdot \omega_{y} \omega_{x}$;

$\dot{\vartheta}=\omega_{y} \cdot \sin \gamma+\omega_{z} \cdot \cos \gamma$

$-m \cdot V_{a}\left(\dot{\psi}_{a} \cos \vartheta_{a} \cos \gamma_{a}+\dot{\vartheta}_{a} \sin \dot{\gamma}_{a}\right)=Z_{a}-P \cos \alpha \sin \beta+G \cos \vartheta_{a} \sin \gamma_{a} ;$

$\dot{\psi}=\left(\omega_{y} \cdot \cos \gamma-\omega_{z} \cdot \sin \gamma\right) \cdot \sec \vartheta$

$\dot{\omega}_{y} I_{y}=M_{y}-\left(I_{x}-I_{z}\right) \cdot \omega_{x} \omega_{z}$;

$\dot{\omega}_{x} I_{x}=M_{x}-\left(I_{z}-I_{y}\right) \cdot \omega_{z} \omega_{y}$;

$\dot{Y}=\dot{H}=V \sin \theta$

$\dot{Z}=-V \cos \theta \sin \Psi$;

$\dot{\gamma}=\omega_{x}+\operatorname{tg} \vartheta \cdot\left(\omega_{z} \cdot \sin \gamma-\omega_{y} \cdot \cos \gamma\right)$,

где $V_{a}$ - воздушная скорость центра масс ракеты;

$V$ - скорость движения ракеты,

$P$ - тяга маршевого двигателя,

$m$ - масса ракеты,

$\alpha-$ угол атаки, $\beta$ - угол скольжения,

$X_{a}$ - сила лобового сопротивления,

$g$ - ускорение свободного падения,

$Z_{a}$ - боковая сила, $Y_{a}$ - подъемная сила, $\theta$ - угол наклона траектории,

$\Psi$ - угол пути, $I_{x}, I_{y}, I_{z}$ - осевые моменты инерции,

$\vartheta_{a}, \psi_{a}, \gamma_{a}-$ скоростные углы тангажа, рысканья, крена,

$\omega_{x}, \omega_{y}, \omega_{z}-$ проекции угловой скорости вращения ракеты на оси связанной системы координат,

$M_{x}, M_{y}, M_{z}$ - аэродинамические моменты, $\mathrm{G}$ - сила тяжести.

Из уравнений (1) получим соотношения для нахождения балансировочных для горизонтального полета значений угла атаки и положения руля $\delta_{\beta}$ :

$$
\left\{\begin{array}{l}
P \cdot \alpha+Y_{a}-G \cdot \cos \theta=0 \\
M_{z}=I_{z} \cdot \dot{\omega}_{z}=0
\end{array}\right.
$$

где $Y_{a}=c_{y} \cdot \frac{\rho \cdot V_{a}^{2}}{2} \cdot S ; M_{z}=m_{z} \cdot \frac{\rho \cdot V_{a}^{2}}{2} \cdot S \cdot b_{a} ;$ коэффициент подъемной силы $c_{y}=K_{2} \cdot V_{a} \cdot \alpha+B_{2} \cdot \alpha+K_{\delta} \delta_{b}^{2}-B_{\delta} \delta_{b}$ 
коэффициент продольного момента

$$
\begin{aligned}
& m_{z}=K_{4} \cdot V_{a} \cdot \delta_{s}^{2}+B_{4} \cdot \delta_{\theta}^{2}+K_{5} \cdot V_{a} \cdot \delta_{в}+B_{5} \cdot \delta_{\text {s }}+K_{3} \cdot V_{a} \cdot \alpha+B_{3} \cdot \alpha ; \\
& b_{a}-\quad \text { средняя } \quad \text { аэродинамическая } \quad \text { хорда; } \quad K_{\delta}, B_{\delta},
\end{aligned}
$$

$K_{2}, B_{2}, K_{3}, B_{3}, K_{4}, B_{4}, K_{5}, B_{5}$ - постоянные коэффициенты;

$\rho$ - плотность воздуха на текущей высоте полета,

$S$ - характеристическая площадь ракеты.

После подстановки выражений для сил и моментов при $\alpha=\alpha_{\text {бал }}$, $\delta_{в}=\delta_{\text {бал }}$ соотношения (2) примут вид:

$$
\left\{\begin{array}{l}
K_{\delta} \rho V_{a}^{2} \cdot S \cdot \delta_{\text {бал }}^{2}-B_{\delta} \cdot \rho V_{a}{ }^{2} S \cdot \delta_{\text {бал }}+\left[\left(K_{2} \cdot V_{a}+B_{2}\right) \rho V_{a}^{2} \cdot S+2 P\right] \cdot \alpha_{\text {бал }}=2 m \cdot g \\
\left(K_{4} \cdot V_{a}+B_{4}\right) \cdot \delta_{\text {бал }}^{2}+\left(K_{5} \cdot V_{a}+B_{5}\right) \cdot \delta_{\text {бал }}+\left(K_{3} \cdot V_{a}+B_{3}\right) \cdot \alpha_{\text {бал }}=0 .
\end{array}\right.
$$

Решая систему (3) с двумя неизвестными можно получить значения балансировочного угла атаки $\alpha_{\text {бал }}$ и отклонения рулей $\delta_{\text {бал }}$.

Для синтеза законов управления систему (1) необходимо линеаризовать в окрестности балансировочных значений параметров полета и представить в нормальной форме Коши:

$$
\dot{X}=A X+B U
$$

где $A$ - матрица состояния; $B$ - матрица управления; вектор состояния

$$
X=\left(\begin{array}{ccccccccccc}
\Delta V & \Delta \theta & \Delta \omega_{z} & \Delta \vartheta & \Delta \Psi & \Delta \psi & \Delta \omega_{y} & \Delta \omega_{x} & \Delta Y & \Delta Z & \Delta \psi
\end{array}\right)^{T} ;
$$

вектор управления $U=\left[\begin{array}{c}\Delta \delta \varepsilon \\ \Delta \delta_{n}\end{array}\right] ; \quad \delta 8-$ угол отклонения руля высоты, $\delta_{n}$ - угол отклонения руля направления. Символами $\Delta$ обозначены отклонения соответствующих параметров движения ракеты от программных значений на траектории полета.

\section{Синтез оптимального регулятора}

При относительно небольшой стартовой скорости полета (35 м/с) боковые порывы ветра более 5 м/с способны отклонить ракету от заданной траектории (рис. 1) так, что после включения двигателя она выйдет за пределы луча. В таких условиях на дистанции появления лазерного луча (60 м, 0,9 c) боковое отклонение снаряда составляет 5 м. При этом половина ширины луча составляет всего 3 м. 


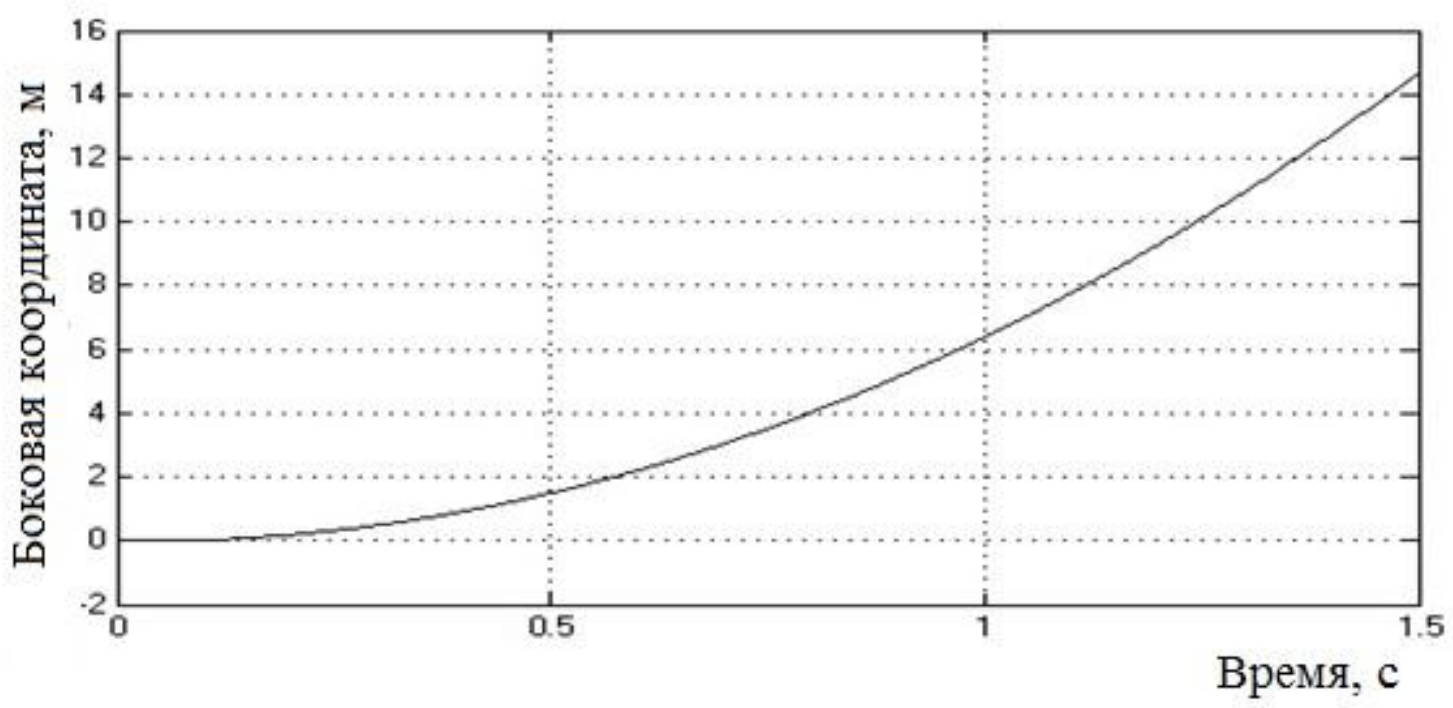

Рис. 1. Траектория движения ракеты при боковом ветре $5 \mathrm{~m} / \mathrm{c}$

Закон управления снарядом для парирования ветровых возмущений найдем на основе теории аналитического конструирования оптимальных регуляторов [3], [4]. Для линейного объекта управления (4) оптимальным в смысле минимума интегрально-квадратичного критерия $I=\int_{0}^{\infty} X^{T} Q X d t+\int_{0}^{\infty} U^{T} R U d t \quad$ будет управление $U=-K X$, где $K=P B^{T} R^{-1}$, а $P$ находится из уравнения Риккати:

$$
P A+A^{T} P-P B R^{-1} B^{T} P+Q=0 ;
$$

$Q, R$-матрицы весовых коэффициентов.

Таким образом, закон управления для каждого из рулей представляет собой линейную комбинацию всех переменных состояния (5):

$$
\begin{aligned}
\delta_{b} & =-\left(K_{V}^{b} \Delta V+K_{\theta}^{b} \Delta \theta+K_{\omega y}^{b} \Delta \omega_{y}+K_{\vartheta}^{b} \Delta \vartheta+K_{\omega z}^{b} \Delta \omega_{z}+K_{\psi}^{\beta} \Delta \psi+K_{\Psi}^{b} \Delta \Psi+K_{\omega x}^{b} \Delta \omega_{x}+\right. \\
& \left.+K_{\gamma}^{b} \Delta \gamma+K_{H}^{b} \Delta H+K_{Z}^{b} \Delta Z\right) ; \\
\delta_{n} & =-\left(K_{V}^{n} \Delta V+K_{\theta}^{n} \Delta \theta+K_{\omega y}^{n} \Delta \omega_{y}+K_{\vartheta}^{n} \Delta \vartheta+K_{\omega z}^{n} \Delta \omega_{z}+K_{\psi}^{n} \Delta \psi+K_{\Psi}^{n} \Delta \Psi+K_{\omega x}^{n} \Delta \omega_{x}+\right. \\
& \left.+K_{\gamma}^{n} \Delta \gamma+K_{H}^{n} \Delta H+K_{Z}^{n} \Delta Z\right),
\end{aligned}
$$

где $K_{i}^{j}$ - элементы $i$-того столбца и $j$-той строки матрицы $K$.

Использование закона управления (6) позволяет снаряду попасть в луч при скорости ветра до 15 м/с (рис. 2). 


\section{Інформаційнісистеми, механіка такерування}

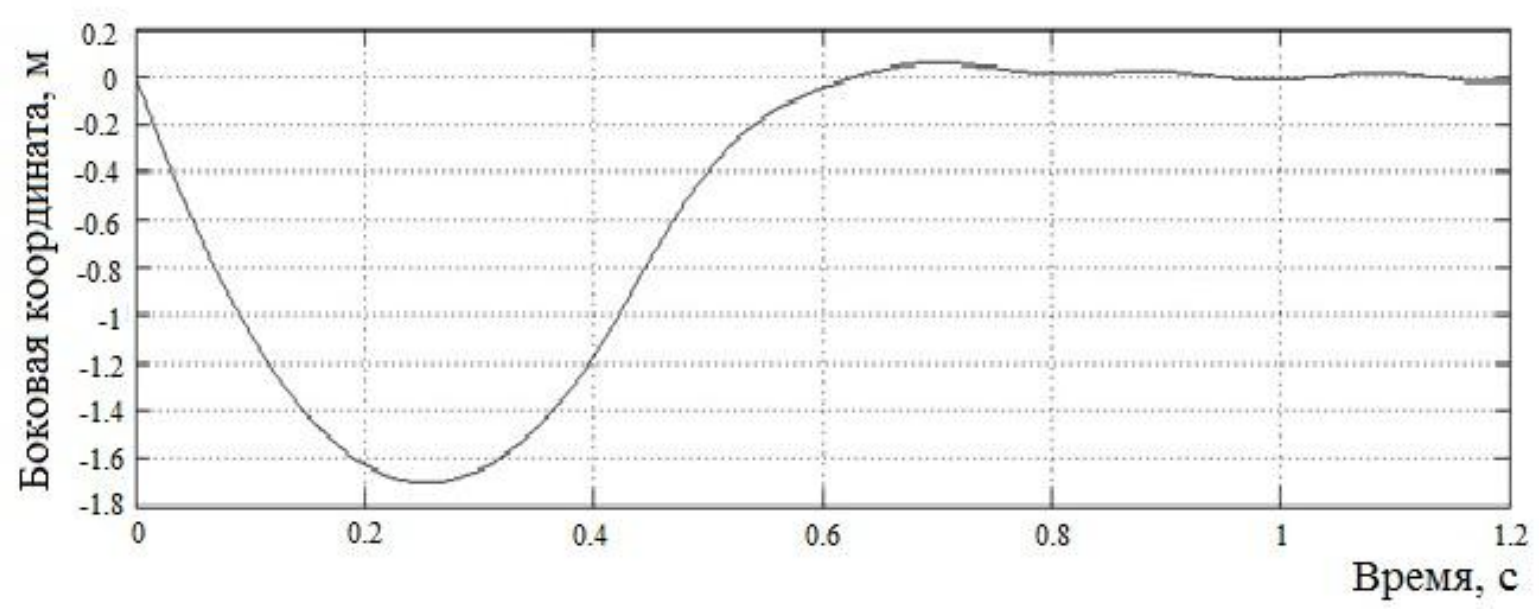

Рис. 2. Траектория движения управляемой ракеты при боковом ветре $14,8 \mathrm{~m} / \mathrm{c}$

Однако для реализации такого регулятора на ракете необходимо использовать полноценную БИНС.

Рассмотрим более простые законы управления:

$$
\begin{aligned}
& \delta_{b}=-\left(K_{\omega y}^{b} \Delta \omega y+K_{\vartheta}^{b} \Delta \vartheta+K_{\omega z}^{b} \Delta \omega_{z}+K_{\psi}^{b} \Delta \psi+K_{\omega x}^{b} \Delta \omega_{x}+K_{\gamma}^{b} \Delta \gamma\right) \\
& \delta_{n}=-\left(K_{\omega y}^{n} \Delta \omega y+K_{\vartheta}^{n} \Delta \vartheta+K_{\omega z}^{n} \Delta \omega_{z}+K_{\psi}^{n} \Delta \psi+K_{\omega x}^{n} \Delta \omega_{x}+K_{\gamma}^{n} \Delta \gamma\right) .
\end{aligned}
$$

Их реализация предполагает измерение только углов ориентации и угловых скоростей снаряда. Для этого нужно иметь на борту трехосный магнитометр и микромеханический датчик угловых скоростей. Такой закон управления может оказаться эффективным, если при действии ветра основной вклад в отклонение ракеты от траектории на дистанции появления луча вносит изменение ее ориентации. Однако имитационное моделирование полета показало, что в этом случае попасть в луч удается при скорости ветра $\leq 7,1 \mathrm{M} / \mathrm{c}$ (рис. 3).

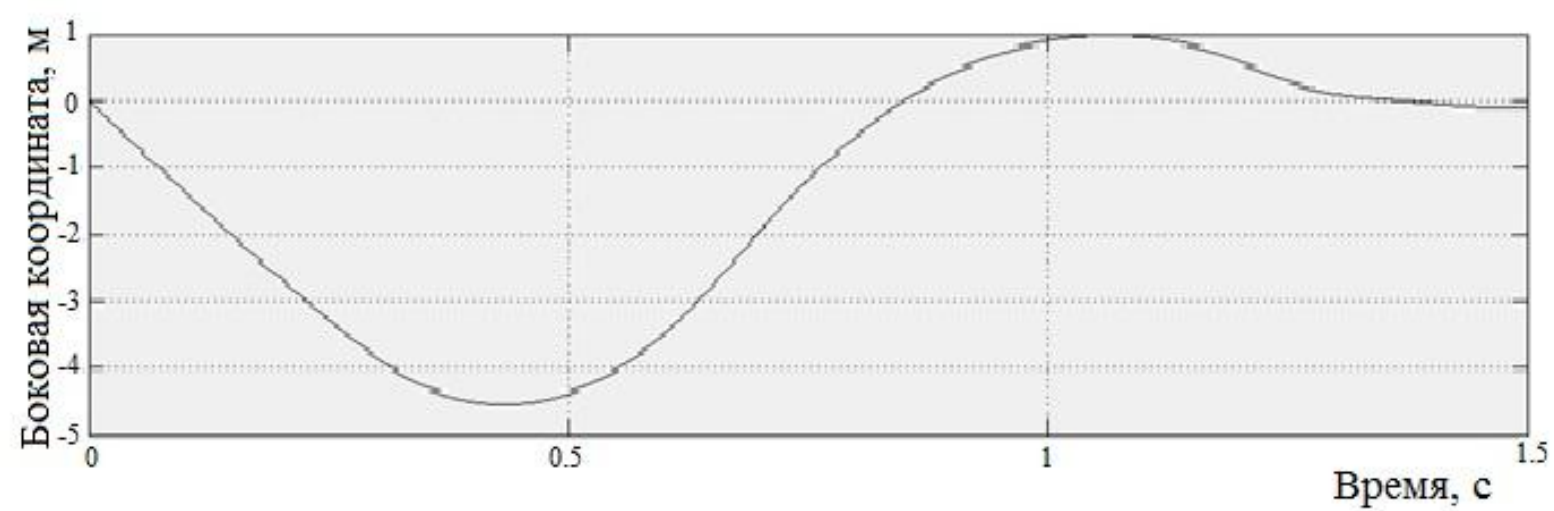

Рис. 3. Траектория движения управляемой ракеты при ветре 7 м/с 
Если в законы управления (7) добавить ошибки стабилизации угла наклона траектории и угла пути:

$$
\begin{aligned}
\delta_{\theta}= & -\left(K_{\theta}^{b} \Delta \theta+K_{\omega y}^{b} \Delta \omega_{y}+K_{\vartheta}^{\beta} \Delta \vartheta+K_{\omega z}^{\beta} \Delta \omega_{z}+K_{\psi}^{\beta} \Delta \psi+K_{\Psi}^{B} \Delta \Psi+\right. \\
& \left.+K_{\omega x}^{b} \Delta \omega_{x}+K_{\gamma}^{b} \Delta \gamma\right) ; \\
\delta_{n} & =-\left(K_{\theta}^{n} \Delta \theta+K_{\omega y}^{n} \Delta \omega_{y}+K_{\vartheta}^{n} \Delta \vartheta+K_{\omega z}^{n} \Delta \omega_{z}+K_{\psi}^{n} \Delta \psi+K_{\Psi}^{n} \Delta \Psi+\right. \\
& \left.+K_{\omega x}^{n} \Delta \omega_{x}+K_{\gamma}^{n} \Delta \gamma\right),
\end{aligned}
$$

ракета сможет попасть в луч при скоростях ветра, не превышающих 10,3 м/с. Применение закона (8) не позволит упростить состав системы по сравнению с (6). Однако на окончательный выбор того или иного закона управления будет влиять точность используемых измерителей.

Для недопущения потери управления ракетой необходимо ввести передаточные коэффициенты $\Delta K w, \Delta K_{l}$, которые позволят плавно перевести её к управлению в лазерном луче:

$$
\begin{aligned}
& \Delta K w=\frac{t_{l}-K w}{t_{k}} \cdot t_{i}+K w \\
& \Delta K_{l}=\frac{t_{l}+K_{l}}{t_{k}} \cdot t_{i},
\end{aligned}
$$

где $t_{l}$ - время начала отключения автономной системы автоматического управления, $t_{k}$ - продолжительность перехода от автономной системы управления ракеты к лазерно-лучевой системе, $t_{i}$ - текущее время; $K w-$ значение передаточного коэффициента автономной системы управления до включения лазерно-лучевой системы, $K_{l}$ - передаточный коэффициент системы управления в лазерном луче.

Тогда законы управления для определения углов отклонения рулей снаряда примут вид:

$$
\delta=\Delta K_{l} \delta_{l u c h}+\delta_{b a l}+\Delta K w \cdot \delta_{d u s}
$$

$\delta_{\text {luch }}-$ рассчитанное значение угла отклонения руля системой управления по лазерному лучу, $\delta_{b a l}-$ значение балансировочного угла установки руля, $\delta_{d u s}-$ значение угла отклонения руля, рассчитанное автономной системой управления ракеты на этапе вхождения в лазерный луч. 


\section{Определение угла установки ствола}

Для успешного выхода управляемой ракеты на заданную траекторию полета большое значение имеет этап встреливания, следовательно - выбор угла начальной установки ствола. Руководствуясь основами внешней баллистики, можно заключить, что горизонтальное размещение пусковой установки не обеспечивает успешного встреливания [5]. Под действием собственной силы тяжести во время вылета из ствола возникает так называемое «проседание» снаряда. В случае нулевого начального угла наклона траектории, при включении маршевого двигателя через 0,2 с поле старта, изделию не хватит перегрузки для устранения данного проваливания. Это приведет к столкновению ракеты с земной поверхностью.

Известные аналитические способы определения угла установки ствола учитывают массу, начальную скорость, предполагаемое воздушное сопротивление. При этом не учитывается влияние тяги двигателя и нелинейность аэродинамики на траекторию полета снаряда. Поэтому требуется пристреливание для дальнейшего составления баллистических таблиц [5], [6]. Построенный таким образом формульный расчет не гарантирует нахождение оптимального угла наклона ствола, который обеспечит сбалансированный выход ракеты на заданную траекторию полета. Поставленная задача может быть решена путем итерационного нелинейного моделирования с применением программного алгоритма поиска.

Начальный угол выставки ствола должен быть оптимальным и уникальным по значению для каждого изделия. Подобранный угол меньше необходимого приведет к падению изделия на начальном участке полета. Угол больше оптимального может привести к вылету снаряда из луча управления, либо заваливанию его носовой части, что также повлечет столкновение с земной поверхностью.

На основе анализа траекторий с разными начальными углами наклона ствола было установлено, что на успешность попадания в луч существенно влияют две величины: минимальное проваливание снаряда ${ }_{\text {min }}$ и значение угла тангажа на этом участке траектории ${ }^{\vartheta} H \min$. Оказалось, что для предотвращения столкновения рассматриваемой ракеты с земной поверхностью в этой точке должны выполняться следующие ограничения: $H_{\text {min }} \geq 0.4 \mu,{ }^{\vartheta}{ }_{H \min } \geq 0^{\circ}$.

Разработанный алгоритм был использован также для подбора угла начальной выставки другого изделия с большей массой. В этом случае накладывались похожие ограничения: $H_{\min } \geq 0.6 \mathcal{M}, \vartheta_{H \min } \geq 0^{\circ}$.

Критерием поиска данного алгоритма является минимум ошибки по высоте и вертикальной скорости при ограничениях тангажа и высоты максимального проседания объекта управления: 


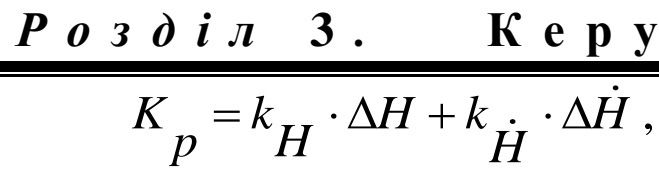

$\Delta H$ - отклонение высоты от заданного значения в момент попадания в луч, $\Delta \dot{H}$ - отклонение вертикальной скорости от заданного значения в момент попадания в луч, $k_{H},{ }_{\dot{H}}$ - весовые коэффициенты. Опытным путем были определены значения весовых коэффициентов для критерия поиска. Для данной модели ракеты критерий (11) примет вид:

$$
K_{p}=0.75 \cdot \Delta H+0.25 \cdot \Delta \dot{H} .
$$

Алгоритм поиска использует метод полного перебора [7]. Он находит все возможные пики максимальных проседаний объекта управления с последующим определением среди них минимального значения. Алгоритм учитывает влияние бокового и вертикального ветра на траекторию полета снаряда. С этой целью поиск углов выставки ствола осуществляется для набора значений скоростей ветра, действующего в разных направлениях.

Траектория движения ракеты и изменения угла тангажа с подобранным в соответствии с (12) начальным углом выставки ствола $4^{\circ} 25^{\prime}$ без ветра изображены на рис. 4. Максимальное отклонение от заданной высоты полета составило 0.5 м, что удовлетворяет заданным условиям, а угол тангажа на этапе проседания снаряда соответствовал заданному условию $\vartheta_{H \min } \geq 0^{\circ}$.
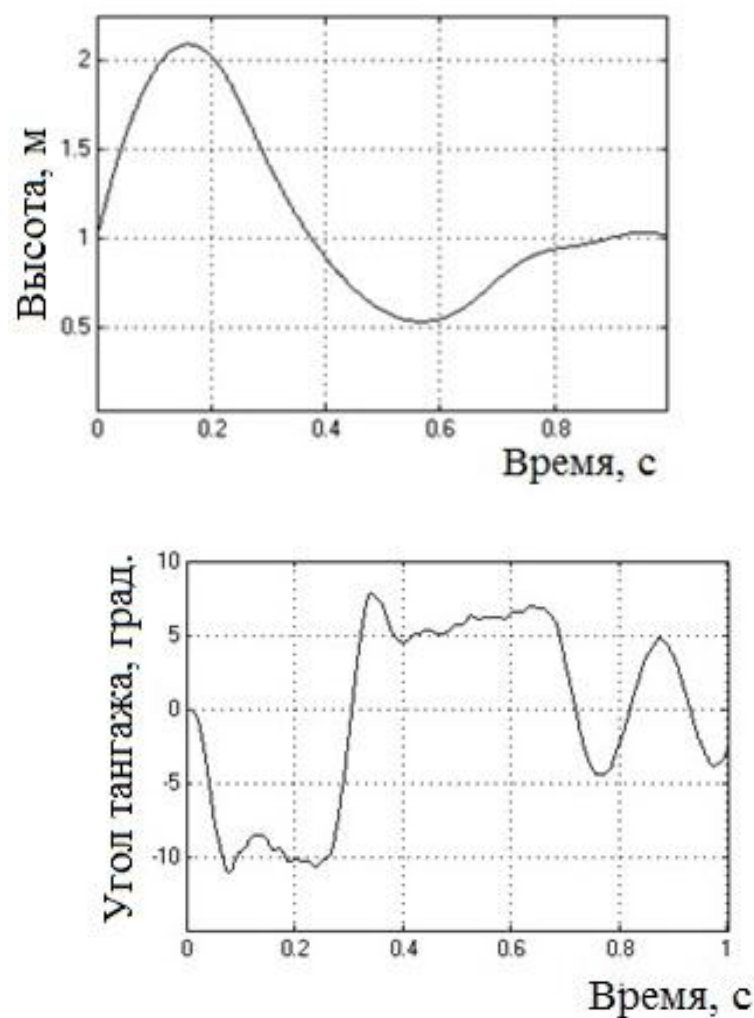

Рис.4. Траектория движения снаряда и изменение угла тангажа после встреливания с начальным углом выставки ствола $4^{\circ} 25^{\prime}$ 
Результаты имитационного моделирования работы системы автоматического управления на этапе вхождения в лазерный луч по закону (8) с углом установки ствола $4^{\circ} 25^{\prime}$ при скорости ветра $10 \mathrm{~m} / \mathrm{c}$ приведены на рис. 5 , рис. 6 .

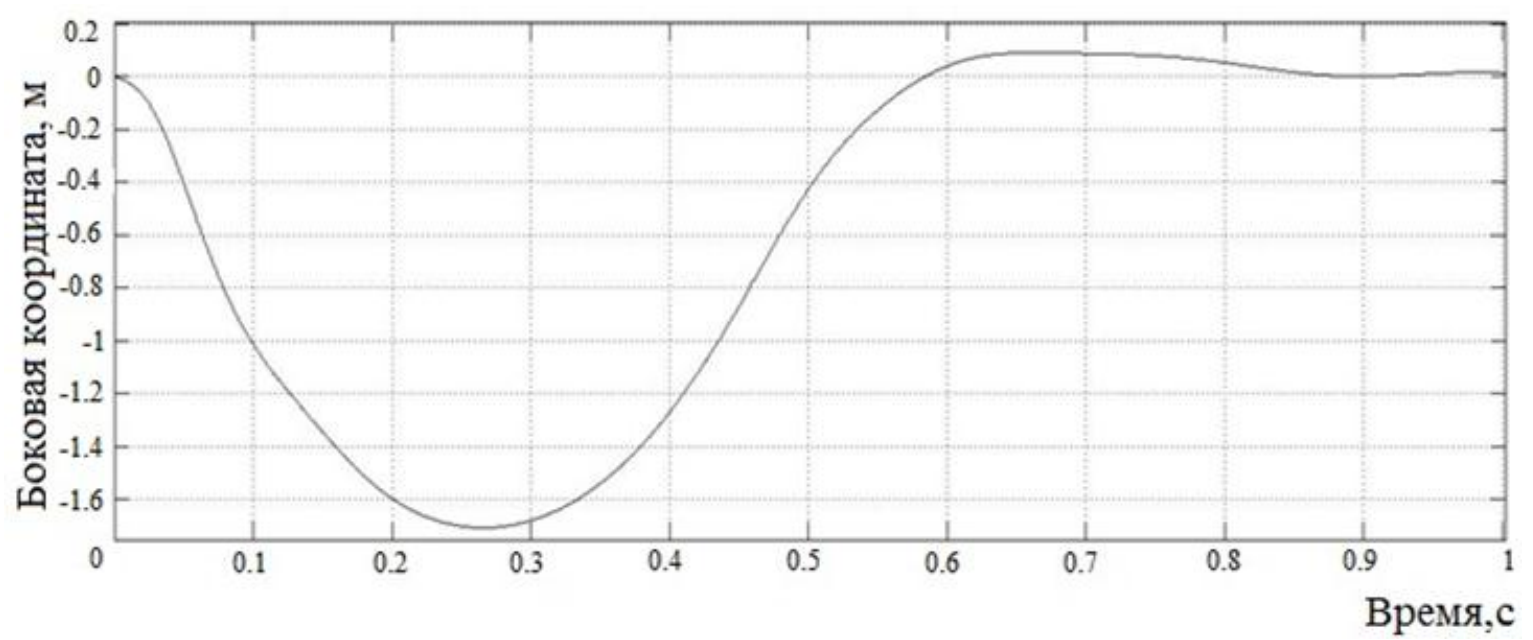

Рис. 5. Траектория полета снаряда в горизонтальной плоскости

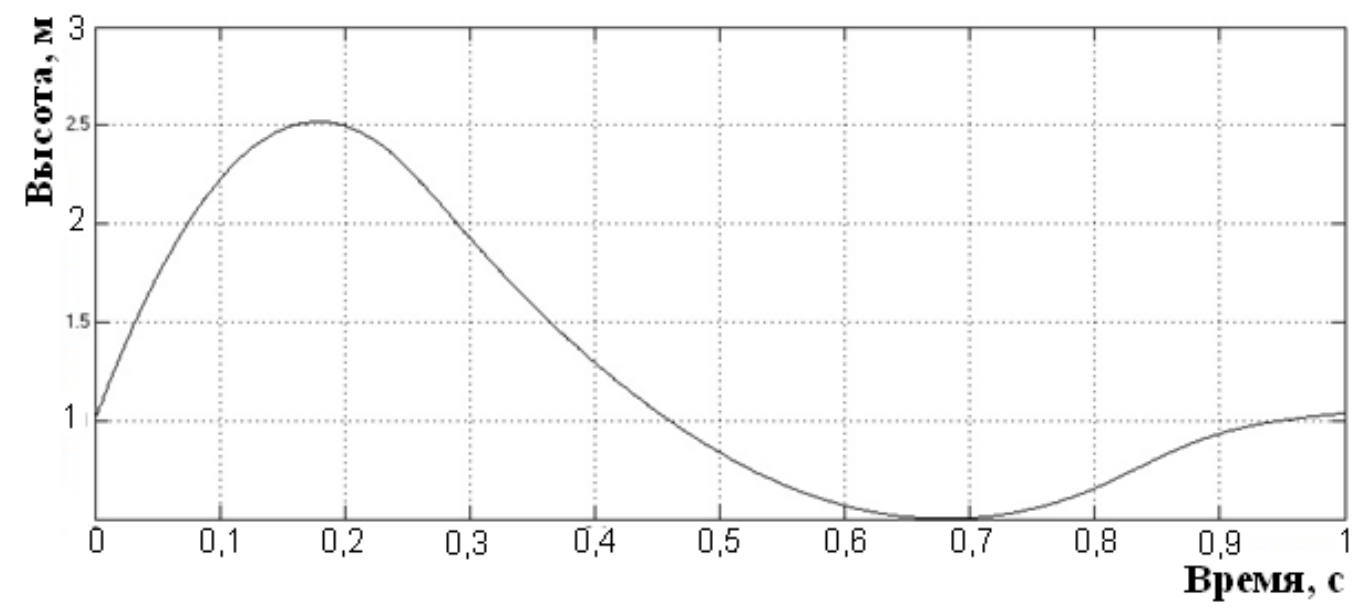

Рис. 6. Траектория полета снаряда в вертикальной плоскости

Как видно из рис. 5, рис. 6 система управления успешно парирует ветер 10 м/с, в результате чего ракета успешно входит в лазерный луч.

\section{Выводы}

Разработанная автономная система управления позволяет обеспечить приведение снаряда в лазерный луч при порывах бокового ветра до 15 м/c. При отсутствии управления на первой секунде полета такая ракета будет потеряна при порыве ветра со скоростью более 5 м/с.

Основным элементом системы управления снарядом на этапе вхождения в лазерный луч должна быть БИНС. Упрощение состава чувствительных элементов до системы ориентации значительно уменьшает допустимую при запуске скорость ветра. Исключение из законов управления 


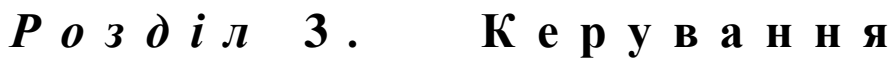

линейных координат и скорости ракеты снижает допустимую скорость ветра до 7,1 м/с. Если в законе управления использовать параметры ориентации и проекции линейной скорости, система может обеспечить попадание ракеты в луч при боковых порывах ветра до 10,3 м/с.

После вхождения снаряда в луч сигналы управления, поступающие на рули от автономной системы, постепенно уменьшаются по разработанному закону. Одновременно пропорционально усиливаются сигналы от лазерно-лучевой системы. Это обеспечивает отсутствие нежелательных переходных процессов при включении лазерно-лучевой системы.

Разработанный алгоритм поиска угла начальной выставки ствола обеспечивает вхождение ракеты в луч на заданной дистанции с минимальными ошибками управления движением в вертикальной плоскости.

\section{Литература}

1. Коростельов О.П. Теоретичні основи проектування ствольних керованих ракет: Монографія. - К.: Книжкове вид-во НАУ, 2007.

2. Лебедев А.А., Чернобровкин Л. С. Динамика полета беспилотных летательных аппаратов. - М.: Машиностроение, 1973., 615 с.

3. Пупков К.А., Егупов Н.Д. Методы классической и современной теории автоматического управления. Теория оптимизации автоматического управления. - М.: Издательство МГТУ им. Н.Э. Баумана, 2004. - Т. 4.$744 \mathrm{c}$.

4. Красовский А.А, Лебедев А.В., Невструев В.В. Теоретические основы пилотажно-навигационных комплексов. - М.: ВВИА им. Жуковського, 1980. -372 c.

5. Дмитриевский А.А., Льсенко Л.Н. Внешняя баллистика. - М.: Москва «Машиностроение», 2005 г., 599 с.

6. Дмитриевский А.А. Внешняя баллистика. - М.: Москва «Машиностроение», 1979 г., 472 с.

7. Е.А. Алёшина, А. В. Пантелеев. Разработка алгоритмического и программного обеспечения метаэвристических методов оптимизации. /Проектно-конструкторские и производственные вопросы создания перспективной авиационной техники // Под ред. проф. Ю. Ю. Комарова. - М.: Изд-во МАИ, 2009. - 424 с. 